\title{
Dietary supplementation of red-osier dogwood polyphenol extract changes the ileal microbiota structure and increases Lactobacillus in a pig model
}

\author{
Shugui Zheng ${ }^{1}$, Jichen Song ${ }^{2}$, Xia Qin ${ }^{1}$, Kai Yang ${ }^{1}$, Mei Liu' ${ }^{1}$, Chengbo Yang ${ }^{2}$ and Charles M. Nyachoti ${ }^{*}$
}

\begin{abstract}
Red-osier dogwood (ROD) extract contains a lot of polyphenols that have the potential for modulation of gut microbiota. However, little information is available about its prebiotic properties. This study investigated the impact of ROD polyphenol extract on the ileal microbiota with dietary supplementation of ROD polyphenol extract in a pig model. The data indicated that supplementation of ROD polyphenol extract significantly increased class Bacilli, order Lactobacillales and family lactobacillaceae. Within family lactobacillaceae, Lactobacillus was the main responder by increasing from 5.92\% to 35.09\%. Further analysis showed that ROD polyphenol extract improved two species Lactobacillus delbrueckii and Lactobacillus mucus. The results of this study suggested that ROD polyphenol extract has the potential to play prebiotic role and confer health benefit through modifying gut microbiota.
\end{abstract}

Keywords: Red-osier dogwood extract, Polyphenols, lleal microbiota, Lactobacillus, Pig

\section{Introduction}

Gut health is essential for overall physical health of humans and animals. There are an enormous number of microbes, most mainly bacteria, in human and animal intestines. Many of these bacteria, which are called commensal bacteria, are highly beneficial. Commensal bacteria play a crucial role in fermenting food, modulating the host immune response, protecting against infections, and regulation of host metabolism, etc. (Flint et al. 2012; Adam et al. 2016). Besides commensal beneficial bacteria, some harmful ones also inhabit the intestine. Actually, it is the balance between beneficial and harmful bacteria that determine the function of gut microbiota and thus health in general (Kevin et al. 2005).

\footnotetext{
*Correspondence: Martin_Nyachoti@umanitoba.ca

2 Department of Animal Science, University of Manitoba, Winnipeg, MB R3T 2N2, Canada

Full list of author information is available at the end of the article
}

Some natural substances can regulate intestinal microbial balance and promote the growth and proliferation of beneficial bacteria. By increasing the number and activity of commensal beneficial bacteria such as Lactobacillus and Bifidobacterium, these substances play an important role in promoting gut health (Ozdal et al. 2016). Redosier dogwood (ROD, Cornus sericea), also called red twig dogwood, red-stem dogwood or red willow, etc., is a common native shrub mainly throughout western and northern America. It grows abundantly in the low wetlands, pastures and marginal land (Isaak et al. 2013). The plant is rich in bioactive substances such as polyphenols, which have remarkable biological activities such as antioxidant and anti-inflammatory properties. Besides, many studies have also shown that plant extracts rich in polyphenols might be used as prebiotics to regulate gut microbiota (Makkar et al. 2007; Fernando et al. 2015).

Red-osier dogwood has long been demonstrated to be effective to treat diarrhea and fever and used as native traditional medicine by the natives of North American. 
Feeding ROD polyphenol extract was able to reduce the incidence of diarrhea and mortality in some animal model and has been suggested to be an alternative to antibiotics used in the feed industry (Schafer et al. 2011). However, the effect of ROD polyphenol extract on the composition of gut microbiota is not well defined. Therefore, the purposes of this study were to explore the effect of dietary ROD polyphenol extract on gut microbial composition in a pig model and deepen the understanding of mechanism by which ROD polyphenol extract plays a positive effect on intestinal microbes. Moreover, in view of the anatomical, physiological and polyphagous similarities between pigs and humans, pig has been considered an ideal model to study intestinal microbiota in humans. Hence, the result of this study may help to elucidate the effect of ROD polyphenol extract on gut microbiota in humans.

\section{Materials and methods}

\section{Characterization of ROD polyphenol extract}

The ROD polyphenol extract was kindly donated by Red Dog Enterprise Ltd. (Winnipeg, Canada). After being defatted with hexanes and extracted as described by Isaaak et al. (2013), polyphenols content was determined by an HPLC technique according to Chen et al. (2016)

Gallic acid and methyl gallate were calibrated at a wavelength of $280 \mathrm{~nm}$, but ellagic acid and quercetin were at $370 \mathrm{~nm}$.

\section{Animals, diets and sampling}

The animal experimental procedures were reviewed and approved by the University of Manitoba Animal Care Committee. All pigs were cared for according to the guidelines of the Canadian Council on Animal Care (CCAC, 2009).

A total of ten crossbred barrows (Duroc $\times$ Landrace $\times$ Large white) from Glenlea Swine Research Unit, University of Manitoba, were selected and surgically fitted with a T-cannula at the distal ileum as previously described (Wubben et al. 2001). After surgery, all the pigs were housed individually under a comfortable environment. Each pen measured $1.2 \times 1.5 \mathrm{~m}$ was equipped with a stainless steel self-feeder and low-pressure nipple drinkers. After adaption period, the pigs with the average body weight of $88.9 \pm 4.3 \mathrm{~kg}$ were randomly assigned to two groups including the control and treatment group with five pigs per group. A non-medicated basal diet containing $73.5 \%$ corn and $23.0 \%$ soybean meal on an air-dry basis as energy and protein sources, respectively, were formulated to meet the NRC (2012) nutrient requirements for the control group. An experimental diet for ROD polyphenol extract group was also prepared with adding $0.5 \%$ ROD polyphenol extract to replace equal amount of corn in basal diet. The ingredients and nutrients of both diets were presented in Table 1. During the 15-day experiment, the pigs were fed twice daily and individually weighed on day 0 and day 15 to calculate average daily gain (ADG). The amount of feed offered and refusals was recorded every day to determine average daily feed intake (ADFI) and then feed to gain ratio (G/F). Ileal digesta samples were collected via ileal fistula after morning feeding on day 15 . Samples were divided into three subsamples and transferred to sterile sample bags. One sub-sample of the digesta was used to measure $\mathrm{pH}$ immediately. The second sub-sample of the digesta was kept on ice and then transferred to $-20{ }^{\circ} \mathrm{C}$ until used for

Table 1 Composition and nutritional contents of control and ROD polyphenol extract supplemented diets

\begin{tabular}{|c|c|c|}
\hline & Control & ROD group \\
\hline \multicolumn{3}{|l|}{ Composition (\%) } \\
\hline Corn & 73.5 & 73.0 \\
\hline Soybean meal (44\%) & 23.0 & 23.0 \\
\hline Vegetable oil & 1.00 & 1.00 \\
\hline ROD polyphenol extract & 0.00 & 0.50 \\
\hline Monocal P (Biofos) & 0.50 & 0.50 \\
\hline Limestone & 0.70 & 0.70 \\
\hline lodized Salt & 0.30 & 0.30 \\
\hline Vitamin-mineral premix & 1.00 & 1.00 \\
\hline \multicolumn{3}{|l|}{ Chemical analysis } \\
\hline DE (Kcal/kg) & 3.48 & 3.47 \\
\hline ME (Kcal/kg) & 3.33 & 3.32 \\
\hline $\mathrm{NE}(\mathrm{Kcal} / \mathrm{kg})$ & 2.45 & 2.44 \\
\hline Crude protein (\%) & 16.2 & 16.2 \\
\hline $\mathrm{Ca}(\%)$ & 0.52 & 0.52 \\
\hline Total P (\%) & 0.49 & 0.49 \\
\hline Av. P (\%) & 0.18 & 0.18 \\
\hline SID Lys (\%) & 0.73 & 0.73 \\
\hline SID Met (\%) & 0.24 & 0.24 \\
\hline SIDThr (\%) & 0.51 & 0.51 \\
\hline SIDTrp (\%) & 0.16 & 0.16 \\
\hline Total polyphenols (mg/kg) & - & 229.56 \\
\hline Gallic acid (mg/kg) & - & 29.78 \\
\hline Methyl gallate (mg/kg) & - & 182.70 \\
\hline Ellagic acid (mg/kg) & - & 17.08 \\
\hline Qercetin (mg/kg) & - & ND \\
\hline
\end{tabular}

Values of nutrient level in the table are calculated values

ROD polyphenol extract, red-osier dogwood polyphenol extract; $D E$, digestible energy; ME, metabolizable energy; NE, net energy; Av. P, available phosphorus; SID, standard ileal digestibility; ND, not detected

Vitamin-mineral Premix provided per kilogram of complete diet: vitamin $\mathrm{A}$ $1300 \mathrm{IU}$; vitamin $\mathrm{D}_{3} 150 \mathrm{IU}$; vitamin $\mathrm{E} 11 \mathrm{IU}$; vitamin $\mathrm{K} 0.5 \mathrm{mg}$; vitamin $\mathrm{B}_{1} 1 \mathrm{mg}$; vitamin $B_{2} 2 \mathrm{mg}$; vitamin $B_{6} 1 \mathrm{mg}$; vitamin $B_{12} 0.005 \mathrm{mg}$; pantothenic acid $7 \mathrm{mg}$; niacin 30mg; folic acid $0.3 \mathrm{mg}$; biotin $0.05 \mathrm{mg}$; copper $3 \mathrm{mg}$; iron $40 \mathrm{mg}$; zinc $50 \mathrm{mg}$; manganese $2 \mathrm{mg}$; selenium $0.15 \mathrm{mg}$; iodine $0.14 \mathrm{mg}$ 
analysis of volatile fatty acids (VFA), and the third subsample was immediately frozen in liquid nitrogen and transferred to $-80{ }^{\circ} \mathrm{C}$ for DNA extraction and microbial analyses.

\section{Metabolites and $\mathrm{pH}$ analysis of ileal digesta}

The concentrations VFAs including acetate, propionate, butyrate, valerate, isovalerate and total VFA in ileal digesta were analyzed by gas chromatography as previously described (Wang et al. 2011). Ileal digesta $\mathrm{pH}$ was measured by a $\mathrm{pH}$ electrode connected to a $\mathrm{pH}$ meter.

\section{Microbial DNA extraction, sequencing and data analysis}

Microbial genomic DNA was extracted from each digesta sample using a commercial DNA isolation kit (Omega Biotek, Norcross, GA, USA) according to the manufacturer's instruction. DNA was quantified using a NanoDrop (Thermo Fisher Scientific, Waltham, MA, United States). The V4-V5 hypervariable regions of bacterial 16S rRNA gene were used for sequencing. After PCR amplification, amplicons were purified using Axy Prep DNA Gel Extraction Kit (Axygen Biosciences, Union City, CA, USA). After that, the purified amplicons were pooled in equimolar and paired-end sequenced on an Illumina MiSeq platform according to the standard protocols.

The sequence data were analyzed using the QIIME (version1.9.1) software package according to the standard guideline. Paired end reads were merged by FLASH (version 1.2.11) with a maximum mismatch rate of 0.10 and the minimum overlap of $10 \mathrm{bp}$. Quality filtering was carried out and sequences were removed if their quality score was smaller than 20 , their overlapped length is less than $10 \mathrm{bp}$ and they contained ambiguous bases, chimeric sequences or a mismatch to primer sequences or barcode tags. The high-quality sequences were clustered into operational taxonomic units (OTUs) at a similarity of $97 \%$. The taxonomy assignment of OTUs was determined by comparing sequences to the Green-gene. The Venn diagrams were constructed to present unique and shared fecal OTUs between control and ROD polyphenol extract groups. $\alpha$-diversity indices, including Shannon index, Simpson index, Chaol, and Observed species (Obs), were calculated using Mothur version 1.39.5 (Patrick Schloss, Ann Arbor, USA). $\beta$-diversity was presented using non-metric multi-dimensional scaling (NMDS) based on Bray-Curtis distances using the GUniFrac packages. Linear discriminant analysis effect size was performed to determine the significantly differential bacteria between two groups. Gene prediction was conducted using PICRUSt 2.
The 16S rRNA sequence information in this study has been deposited into the Sequence Read Archive (SRA) database under accession no. PRJNA692786.

\section{Statistical analysis}

All statistical analysis in this research was carried out in $\mathrm{R}$ 3.2.1. Welch'st-test between-group $\alpha$-diversity comparisons was calculated with $\mathrm{R}$. The software was also employed to calculate non-metric multi-dimensional scaling (NMDS) of (un) weighted UniFrac distances. The value of $P<0.05$ was considered statistically significant. Spearman's rank correlation analysis between important bacteria and metabolite profiles was performed using Graphpad Prism version 6.0 (GRAPHPAD Software, San Diego, CA, USA). Correlation was considered significant at $P<0.05$.

\section{Results}

\section{Polyphenol content of ROD polyphenol extract}

The polyphenol extract contained abundant polyphenols, including $5.955 \mathrm{mg} / \mathrm{g}$ of gallic acid, $36.539 \mathrm{mg} / \mathrm{g}$ of ethyl gallate and $3.415 \mathrm{mg} / \mathrm{g}$ of ellagic acid.

\section{Dietary ROD polyphenol extract had no significant effect on performances in finishing pigs}

There was no significant effect on the ADG, ADFI and F/G due to supplementation of ROD polyphenol extract (Table 2).

\section{The effect of dietary supplementation of ROD polyphenol extract on volatile fatty acid production and $\mathrm{pH}$}

To determine the effect of ROD polyphenol extract on the ileal fermentation parameters, the concentrations of acetate, propionate, butyrate, valerate, isovalerate, total VFA and ileal $\mathrm{pH}$ were determined. As shown in Table 3, the concentration of propionate was significantly enhanced by dietary supplementation of ROD polyphenol extract compared with the control group. However, there were no differences in acetate, butyrate, valerate, isovalerate, total VFA and ileal $\mathrm{pH}$ between the control and ROD polyphenol extract group.

Table 2 Effects of ROD polyphenol extract supplementation on growth performances in finishing pigs

\begin{tabular}{lcccl}
\hline & Controls & ROD group & SEM & P value \\
\hline ADG $(\mathrm{g} / \mathrm{d})$ & 1051.6 & 1207.6 & 80.3 & 0.191 \\
$\mathrm{ADFl}(\mathrm{g} / \mathrm{d})$ & 2813.3 & 2813.3 & 0.0 & - \\
F/G & 2.71 & 2.39 & 0.19 & 0.270 \\
\hline
\end{tabular}

ADG, average daily gain; $A D F I$, average daily feed intake; $F / G$, feed to gain ratio; SEM, standard error of the mean

Data are presented as group means of five piglets per group 
Table 3 Effects of dietary ROD polyphenol extract supplementation on VFA concentration and $\mathrm{pH}$ of ileal digesta in finished pigs ( $\mu \mathrm{mol} / \mathrm{g})$

\begin{tabular}{lrrrl}
\hline & Control & ROD group & SEM & P value \\
\hline Acetate & 94.81 & 65.82 & 19.47 & 0.312 \\
Propionate & 3.99 & 11.59 & 2.65 & 0.045 \\
Butyrate & 12.91 & 9.30 & 3.91 & 0.567 \\
Valerate & 0.40 & 1.03 & 0.43 & 0.405 \\
Isovalerate & 1.63 & 1.51 & 0.48 & 0.863 \\
Total VFA & 113.76 & 89.47 & 24.29 & 0.486 \\
pH & 6.78 & 6.66 & 0.13 & 0.582 \\
\hline
\end{tabular}

VFA, volatile fatty acid

SEM, standard error of the mean

\section{Dietary ROD polyphenol extract increased the diversity and numbers of bacteria in ileal digesta}

Sequencing of 16 S rRNA indicated that a total of 578,689 tags were obtained from the ileal samples. The stable plateau of the Shannnon-Wiener curves observed in all the samples indicated that the sequencing data were large enough to provide ample microbial information (Fig. 1A). Shannon index has indicated that dietary supplementation of ROD polyphenol extract significantly increased the $\alpha$-diversity of the bacterial composition (Fig. 1B).
Moreover, a statistically significant difference of bacterial composition between control and ROD polyphenol extract treatments was observed in non-metric multidimensional scaling (NMDS) analysis, a kind of $\beta$-diversity analysis, which was conducted to explore the structural variations of the gut microbiota across the samples using Bray-Curtis distance metrics (Fig. 1C).

The ileal microbiota analysis indicated a total of 321 OUTs were detected at the $97 \%$ identity. Approximately $44 \%$ OTUs was shared between the control and ROD polyphenol extract groups. Venn diagrams indicated that dietary supplementation of ROD polyphenol extract greatly increased the number of unique OTUs (Fig. 1G). This result suggested that the proportions of particular bacteria were enhanced by ROD polyphenol extract treatment.

\section{Dietary ROD polyphenol extract changed ileal digesta microbiota structure}

To further elucidate the effect of dietary supplementation of ROD polyphenol extract on pigs, microbiota structure analysis was performed. The result was showed in Fig. 2. Firmicutes and Proteobacteria were the two top phyla presented in the ileal digesta and accounted for more than $95 \%$ of all phyla in both control and ROD polyphenol extract group. At the class level, the main bacteria

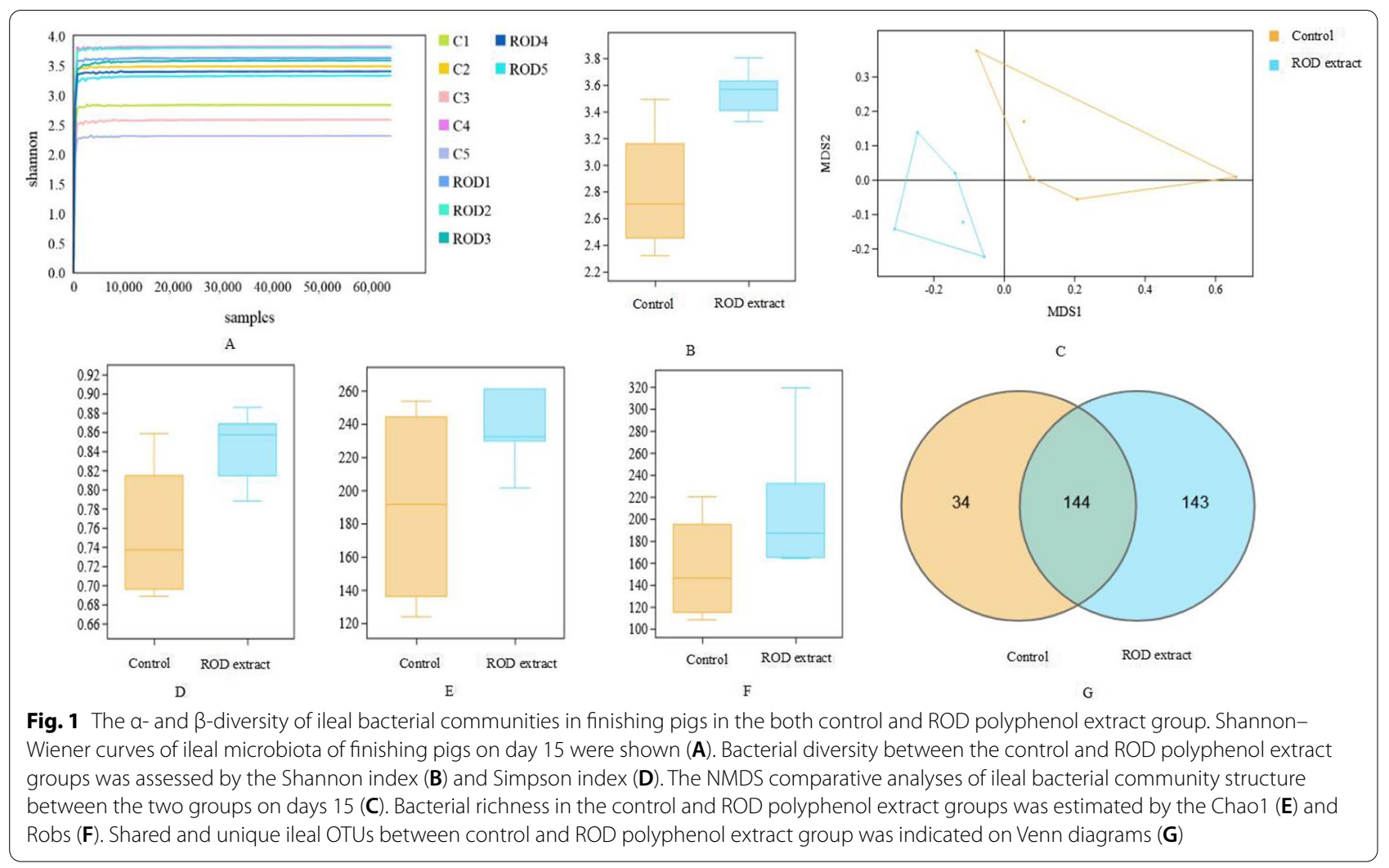



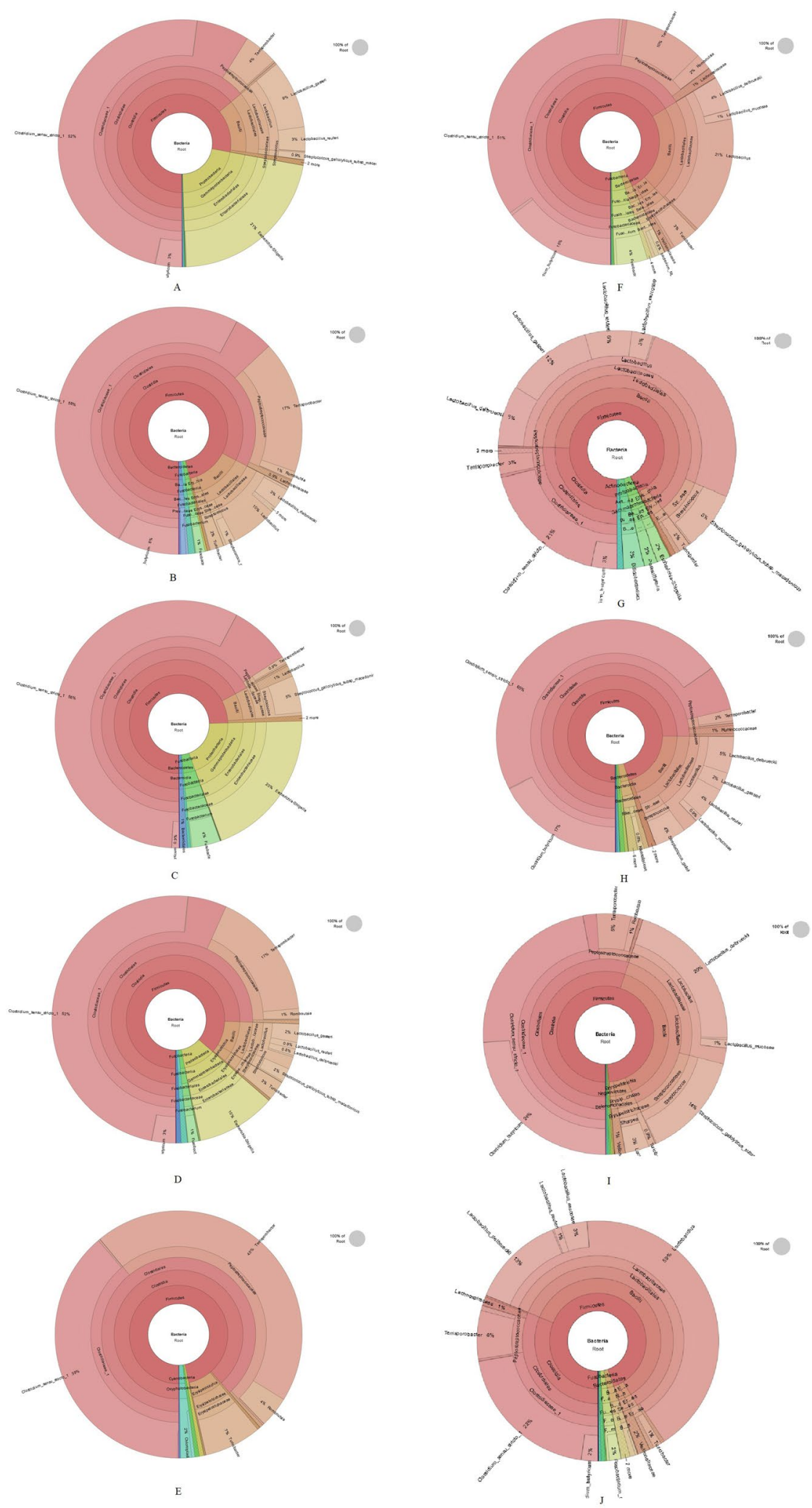

Fig. 2 Krona pie charts of ileal microbial compositions for the five sample in control group (A-E) and the five samples in ROD polyphenol extract group $(\mathbf{F}-\mathbf{J})$. The diagram shows different taxonomic levels of ileal microbial compositions including domain, phylum, class, order, family, genus and species from the center to the outside. The fan-shaped area corresponds to the relative abundance of corresponding microbes 
were Clostridia, Bacilli, and Gammaproteobacteria in control group. But a notable alteration in composition of microbiota was observed due to ROD supplementation in that Bacilli increased dramatically and replaced considerable proportions of Clostridia and Gammaproteobacteria. At the order level, Lactobacillales replace Colstridiales and Enterobacteriales and became significantly higher bacteria in ROD polyphenol extract group compared with the control group in which Colstridiales and Enterobacteriales accounted for greater than 85\% of all orders. Further analysis at family level found that ROD polyphenol extract promoted a striking increase of Lactobacillaceae and replace a considerable proportion of Clostridiaceae_1, Peptostreptococcaceae, and Enterobacteriaceae. At genus level, dietary supplementation of ROD polyphenol extract increased Lactobacillus instead of some of Clostridium_sensu_stricto_1, Terrisporobacter, Escherichia-Shigellais and became prominent bacteria in ROD group compared with the control group.

\section{Dietary ROD polyphenol extract increased the abundance of beneficial bacteria}

Relative abundance analysis showed that order Lactobacillales exclusively occupied class Bacilli and family Lactobacillaceae contained only genus Lactobacillus. Compared with control group, the relative abundance of Bacilli in ROD polyphenol extract group was enhanced by over 5 times from $7.89 \%$ to $40.60 \%$. In the same way, the relative abundance of Lactobacillales in ROD treatment group was also increased by the same number. At family and genus levels, dietary supplementation of ROD polyphenol extract significantly increased the relative abundance of lactobacillaceae by 5.9 times from $5.92 \%$ to $35.09 \%$ compared with the control group. Similarly, the relative abundance of Lactobacillus in ROD polyphenol extract treatment group was more than those in the control group with the same amount (Table 4).

Supplementation of ROD treatment significantly improved Lactobacillus delbrueckii from $0.85 \%$ to
$10.15 \%$ by nearly 12 times and notably increased Lactobacillus mucosae from $0.18 \%$ to $1.94 \%$ by over 10 times, respectively (Table 4 ).

LEfSe analysis was further carried out and discovered the dietary supplementation of ROD polyphenol extract specifically increased the abundance of some beneficial bacteria. The results showed that there was no differential enrichment of bacteria existing in the control group (Fig. 3). However, in the ROD polyphenol extract group, Bacilli, Lactobacillales, Lactobacillaceae, Lactobacillus were the dominant class, order, family and genus, respectively. Two dominant species Lactobacillus_delbrueckii and Lactobacillus_mucosae were also noted. Besides, dominant bacteria also included Family_XIII and two genera sharpea and Dialister as well as a species Lachnospiraceae_ bacterium _ DJF_ LS97k1 (Fig. 3).

\section{Dietary ROD polyphenol extract altered metagenomic metabolic functions of ileal bacteria}

For further understand the effect of ROD polyphenol extract on intestinal bacteria, function predictions were performed using PICRUSt 2. The result shown that a total of 26 gene families were predicted to appear in all samples of both control (Fig. 4A) and ROD polyphenol extract group (Fig. 4B). Metabolomic analysis has indicated that bacteria from ileal digesta includes those involved in carbohydrate and amino acid metabolism, metabolism of cofactors and vitamins, gene replication and repair, cell motility, and membrane transport. Functional difference analysis showed that the ROD polyphenol extract group had a greater relative abundance of gene families involved in biosynthesis of other secondary metabolites $(P<0.05)$. At the same time, the ROD group had a lower relative abundance of gene families relevant to amino acid metabolism, metabolism of cofactor and vitamins, and cell motility $(P<0.05)$ (Fig. 4C).

Table 4 Relative abundances of main different bacteria in ileal digesta at different taxonomic levels between control and ROD treatment group (\%)

\begin{tabular}{llllcc}
\hline Classification levels & Taxonomic names & Control & ROD polyphenol extract & SEM & P-value \\
\hline Phylum & Firmicutes & 86.38 & 94.86 & 3.98 & 7.23 \\
Class & Bacilli & 7.89 & 40.60 & 7.23 & 0.022 \\
Order & Lactobacillales & 7.89 & 40.60 & 35.09 & 7.26 \\
Family & lactobacillaceae & 5.92 & 35.09 & 7.26 & 0.022 \\
Genus & Lactobacillus & 5.92 & 10.15 & 2.22 & 0.033 \\
Species & Lactobacillus delbrueckii & 0.85 & 1.94 & 0.41 & 0.032 \\
& Lactobacillus mucosae & 0.18 & & 0.029 \\
\hline
\end{tabular}




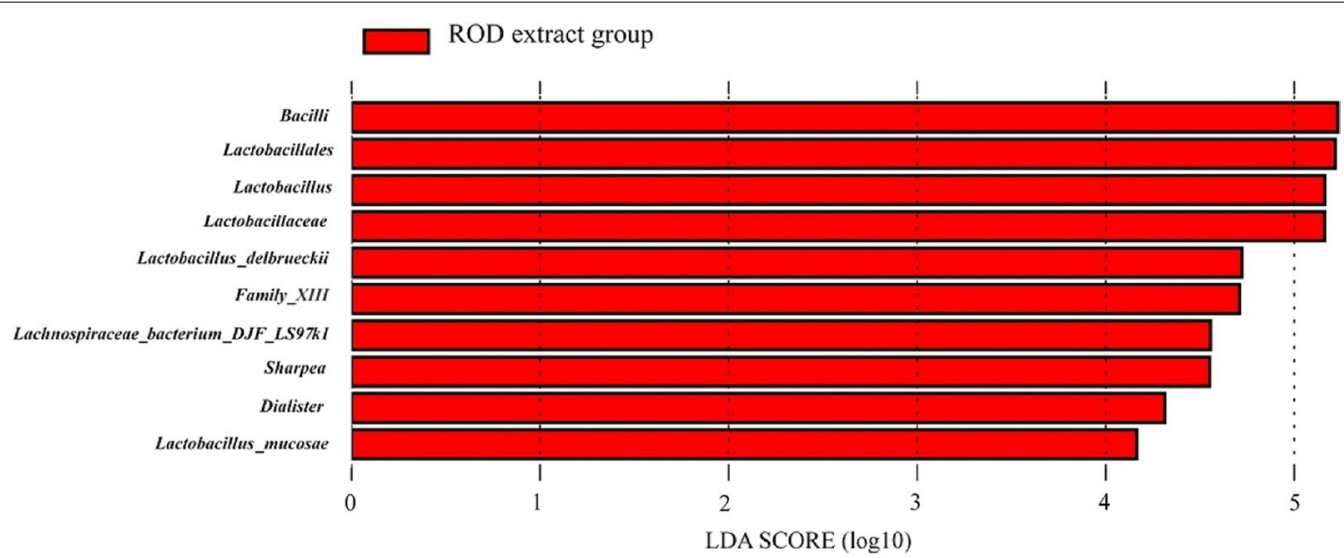

Fig. 3 Differential enrichment of bacteria due to supplementation of ROD polyphenol extract. There is not differential enrichment of bacteria in the control group, and differential enrichment of bacteria in ROD polyphenol extract group is presented with the logarithmic LDA score of 3.0 as the cutoff value

\section{Correlation analysis between gut microbes and different volatile fatty acids, and $\mathrm{pH}$}

To further elucidate whether the intestinal bacterial community has the relation with metabolites in ileal digesta, Pearson correlation analysis was carried out among different VFA, $\mathrm{pH}$ and the bacterial abundance at species level. As showed in Fig. 5, Propionate was positively correlated with Lactobacillus delbrueckii, Streptococcus gallolyticus subsp macedonicus and Lachnospiraceae bacterium DJF LS97k1, respectively. Valerate had obviously positive correlation with Lactobacillus mucosae and Porphyromonas sp 2121, respectively. However, isovalerate presented notably positive correlation with Fusobacterium necrophorum subsp necrophorum, bacterium NLAE-zl-C391, Bacteroides fragilis and Clostridium aurantibutyricum, respectively.

\section{Discussion}

As the need to explore the potential of prebiotics in promoting gut health, increasing attention has been paid to the potential prebiotic effects of plant extracts other than oligosaccharides (Liu et al. 2014; Moorthy et al. 2020). The International Scientific Association for Probiotics and Prebiotics (ISAPP) proposed prebiotic as a substrate selectively utilized by host microorganisms conferring a health benefit (Swanson et al. 2020). This new definition extends the concept of prebiotic to phytochemicals, phenolics, and polyunsaturated fatty acids (Brochot et al. 2019). Studies have demonstrated that there was complicated interaction between phenolics and gut microbiota. On one hand, intestinal microbes could metabolize phenolics. Some bacterial species, such as Lactobacillus sp., Bifidobacterium sp. Bacteroides sp. Eubacterium $s p$., Escherichia coli, etc. could catalyze the phenolics metabolism (Kutschera et al. 2011). Further investigation suggested that by converting into more active biologically phenolic metabolites, Gut microbita's metabolism could enhance the biological activity of phenolics. On the other hand, a number of evidences from in vivo and in vitro studies showed that phenolic metabolites were able to act as prebiotics and modulate the ecology of gut microbiota via promoting the proliferation of beneficial bacteria and inhibiting the growth of pathogenic microbes, consequently influencing the host health (Ozdal et al. 2016).

The complex interaction between phenolics and gut microbiota contributes greatly to the overall health of animals and humans (Gowd et al. 2019). An in vitro research showed that phenolics from green tea, black tea and oolong tea significantly influences gut microbiota by increasing the growth of Lactobacillus spp., Bifidobacterium spp. and Enterococcus spp while inhibiting the growth of Prevotella, Bacteroides, and Clostridium histolyticum groups (Sun et al. 2018).Several experimental evidences also showed that phenolics-rich compounds exerted the antioxidant and anti-inflammatory effects via the interaction with gut microbiota (Pandey et al. 2009; Gowd et al. 2019). In the present study, dietary supplementation of ROD polyphenol extract increased microbial $\alpha$-diversity and the numbers of commensal bacteria. Clustering pattern also showed that ileal digesta samples from ROD supplemented pigs clustered away from the control. These results suggested that ROD polyphenol extract promoted more diverse and complex microbiome in the ileum. A more diverse gut microbiota may help to improve gut health and, in turn, reduce the risk of disease (Flint et al. 2012).

Moreover, dietary supplementation of ROD polyphenol extract changed the ileal microbiota structure and 


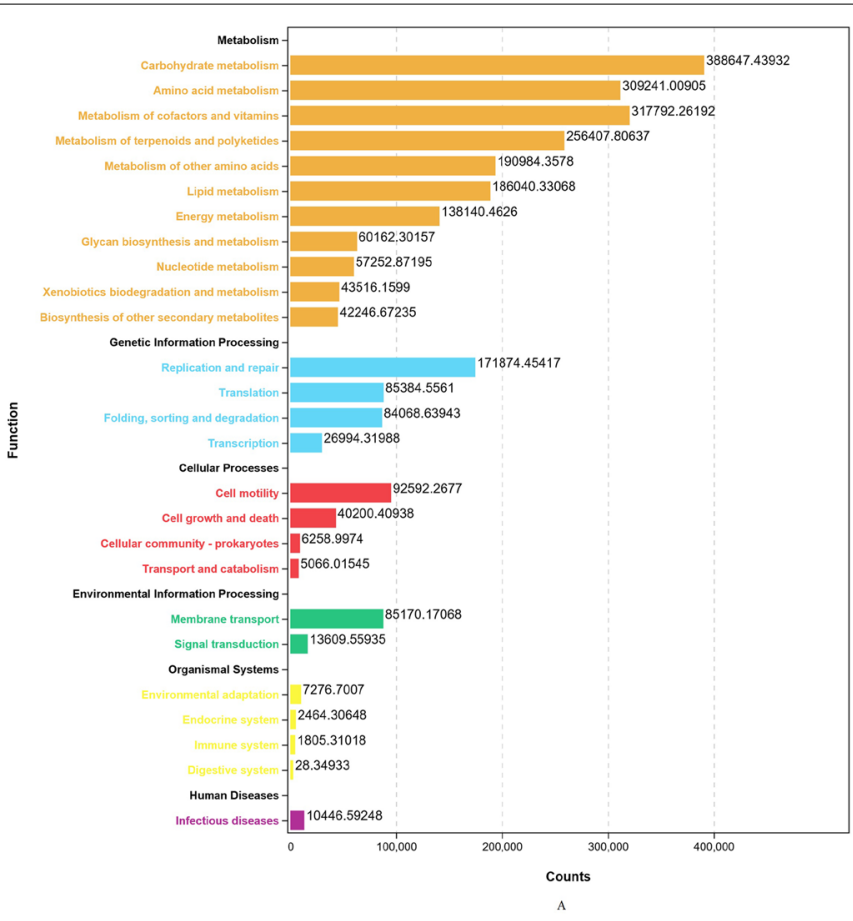

\section{Metabolsm}

1 Genetic Information Processing

- Environmental Information Processing

norganismal Systems

- Human Diseases

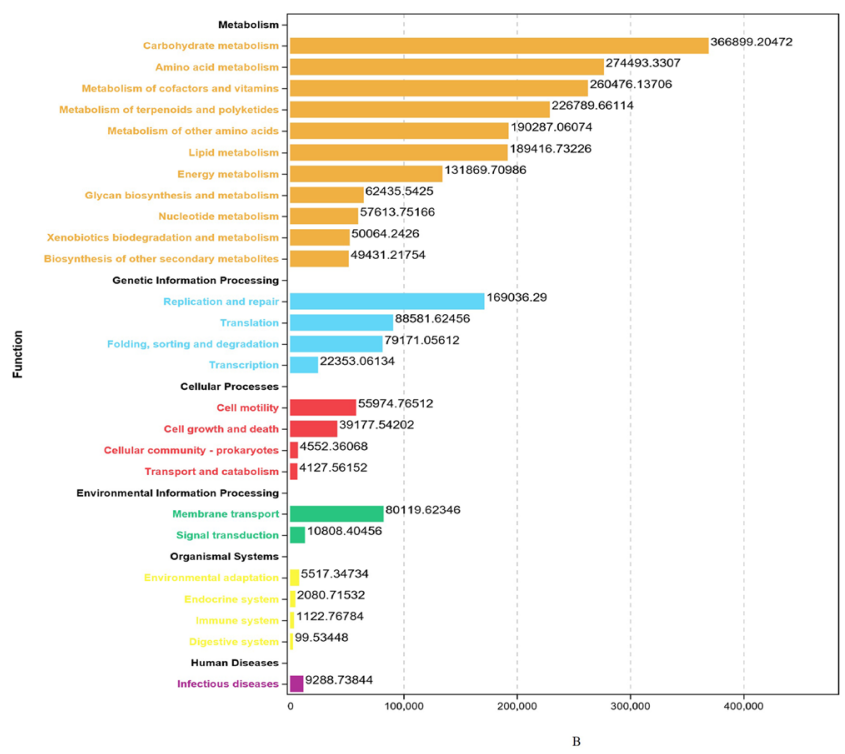

Metabolism Genetic Information Procossing Environmental Information Processing Organismal Systems

Control

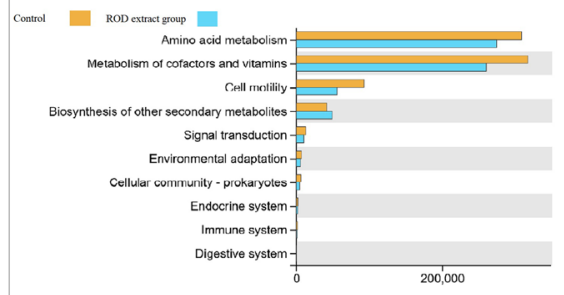

Mean abundance

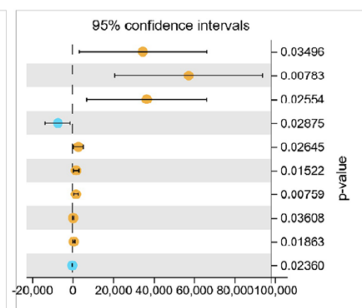

Difference in mean abundance

Fig. 4 Functional diversity of ileal bacterial community of control (A) and ROD polyphenol extract group (B). Function predictions were performed by using PICRUSt 2. Functional difference between control and ROD polyphenol extract group, and only the abundances of KEGG pathways of ileal microbiota that were significantly influenced by ROD polyphenol extract are shown (C) 


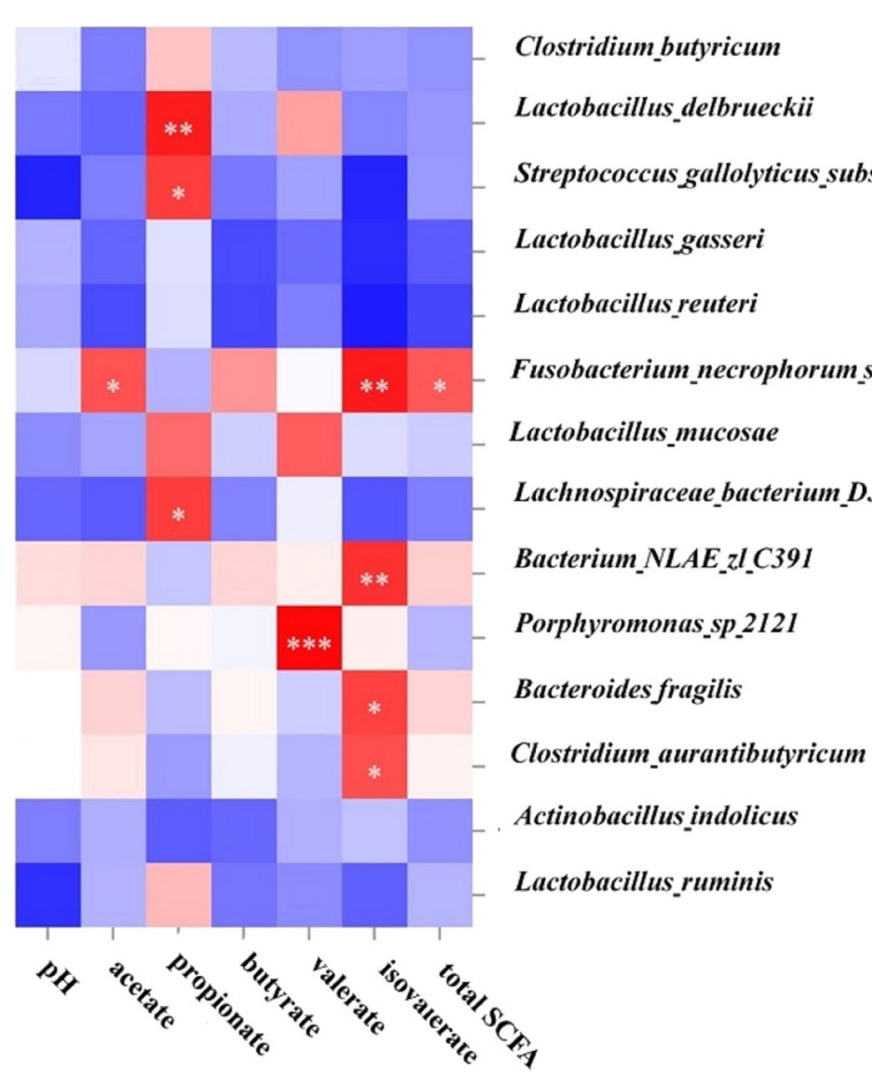

Fig. 5 Pearson correlation analysis between bacteria and the concentration of volatile fatty acids (VFA), pH in ileal digesta of finishing pigs. The cells are colored based on the Pearson's correlation coefficient. Red represents positive correlation. Blue represents negative correlation, and white represents no significant correlation. An asterisk represents significant correlation $(P<0.05)$, and double asterisk represents very significant correlation $(P<0.01)$

increased beneficial bacteria. In the present research, Firmicutes and Proteobacteria were observed to be the top two phyla in both control and ROD polyphenol extract group. This result was consistent with a previous study, where Firmicutes and Proteobacteria were two most dominant phyla in ileal digesta of pigs (Torres-Pitarch et al. 2020). At the class level, the dominant bacteria were Clostridia, Gammaproteobacteria and Bacilli in control group, whereas dietary supplementation of ROD polyphenol extract significantly increased the relative abundance of Bacilli by replacing substantial proportions of Clostridia and Gammaproteobacteria. Lactobacillales was the only order of class Bacilli observed in pigs' ileal digesta in this study. Dietary supplementation of ROD polyphenol extract significantly increased the relative abundance of Lactobacillales and replaced large proportions of the colstridiales and Enterobacteriales, the two orders that accounted for the most of the orders in control group. The order Lactobacillales consists of six families including Lactobacillaceae, Streptococcaceae, Leuconostocaceae, Enterococcaceae, Aerococcaceae, and
Carnobacteriaceae in taxonomy. Among these families, only Lactobacillaceae and Streptococcaceae were found in pigs'ileal digesta in the present study. After dietary supplementation of ROD polyphenol extract for 15 days, the relative abundance of Lactobacillaceae was observed to increase significantly by replacing considerable proportions of Clostridiaceae 1, Peptostreptococcaceae and Enterobacteriaceae in the control group. Family Lactobacillaceae contain only one genus Lactobacillus which is the major beneficial commensal inhabitant in human and animal intestines. Lactobacillus maintains intestinal health by production of antimicrobial substances, preventing gut colonization of enteric pathogenic bacteria (Lee et al. 2012), stimulating the mucosal immune response (Erickson et al. 2000; Hou et al. 2015) and regulating the cytokine expression (Smits et al. 2005; Frossard et al. 2007). The present study showed that dietary supplementation of ROD polyphenol extract significantly increased Lactobacillus by replacing considerable proportions of Clostridium sensu stricto 1, Terrisporobacter and Escherichia Shigellais, suggesting that ROD 
polyphenol extract could optimize the microbiota structure and increased beneficial bacteria.

From the perspective of taxonomy, the genus Lactobacillus includes 152 validly described species (Salvetti et al. 2012). The present study showed that compared with control group dietary supplementation of ROD polyphenol extract significantly increased the abundance of two species Lactobacillus delbrueckii and Lactobacillus mucosae. As type species of the genus Lactobacillus, Lactobacillus delbrueckii has long been demonstrated to have probiotic effect on human and animals (Yu et al. 2012; Lick et al. 2001; Sun et al.2015). It played an important role in maintaining gut health via alleviating intestinal tissue damage (Sun et al. 2015) and renovating mucosal barrier destruction (Yu et al. 2012). It has also anti-inflammatory property (Clarissa et al. 2014; MoroGarcía et al. 2013) and the ability to restore intestinal microbita dysfunction (Sun et al. 2015). Lactobacillus mucosae, however, was first isolated from pig intestines and had mucus-binding activity. It has been shown to decrease epithelial permeability, improve epithelial barrier function and provide competitive exclusion against many of pathogenic bacteria (Watanabe et al. 2010). The results of this study indicated that dietary ROD polyphenol extract increased the abundance of Lactobacillus delbrueckii and Lactobacillus mucosae in pigs'ileal digesta, suggesting the potential of ROD polyphenol extract to modulate gut health and be the promising prebiotics for animal and food industry.

The function of intestinal microbial community is closely related to microbiota structure and usually changes with changes in its composition (Mauro et al. 2013; Cao et al. 2020). In this study, results of PICRUSTs 2 indicated in finishing pigs had a variety of functions, particularly for carbohydrate metabolism, amino acid metabolism, and metabolism of cofactors and vitamins. The systems of carbohydrate metabolism, amino acid metabolism, and cofactors and vitamins metabolism are naturally present in all living cells and is crucial for metabolism of substance and energy in living organism, thereby having great significance for microbial survival. Due to the fact that ROD polyphenol extract was capable of alter gut bacteria composition, intestinal bacterial functions may also be changed. In this study, in comparison with control group, the relative abundance of gene families associated to biosynthesis of other secondary metabolites was increased in the ROD polyphenol extract group. While the relative abundance of gene families related to amino acid metabolism, metabolism of cofactor and vitamins, cell motility was decreased after ROD polyphenol extract treatment. The abundance of genes associated with the biosynthesis of secondary metabolites was increased due to supplementation of ROD polyphenol extract, suggesting that ROD polyphenol extract may have a positive impact on secondary metabolites, which perhaps is associated with the polyphenols in ROD polyphenol extract. The decreased abundance of genes associated with amino acid metabolism indicates that the ability of metabolizing amino acid might be lowered by decreasing the number of amino-acid metabolizing bacteria due to ROD supplementation. However, due to prediction limitation and complex microbial function, further research should be conducted to validate this notion.

As the molecules produced by bacteria when they ferment dietary components primarily carbohydrate inside the intestine, volatile fatty acids (VFA), mainly acetate, propionate and butyrate, are beneficial to the host health when present in sufficient quantities (LeBlanc et al. 2017). In this study, dietary supplementation of ROD polyphenol extract was shown to significantly increase the concentration of ileal propionate. This might result from the change of ileal digesta microbiota structure. We also demonstrated that there was a strong positive correlation between Lactobacillus delbrueckii and propionate in this study. Propionate is one of the most important VFA and it can help improve the host gut health. A research has shown that propionate exerted beneficial effects on the intestinal epithelium by improving intestinal barrier function, inhibiting inflammation, and modulating oxidative stress (Tong et al. 2016). In addition, another research also proved that propionate inhibits Salmonella colonization and expansion in the intestinal tract and whereby protect against Salmonella infections (Jacobson et al. 2018). More than that, propionate-associated health benefits were demonstrated to extend beyond the gut epithelium. Propionate was proved to lower liver lipogenesis, hepatic and plasma cholesterol levels, and carcinogenesis in other tissue. (Nishina et al. 1990; Delzenne et al. 2002; Adam et al. 2001; Jan et al. 2002). Besides, among VFAs, propionate had particularly been considered as a satietyinducing substance with a significant effect on feeding behavior and energy intake (Ruijschop et al. 2008).

Taken together, this study demonstrated that ROD polyphenol extract has the potential to regulate gut microbial diversity, increase the abundance of beneficial bacteria, and promote to form a more balanced gut microbiota structure. Microbial metabolites, particularly propionate, were improved by the ROD polyphenol extract, exerted further beneficial effect in pig model. Our findings show that ROD may be a promising prebiotic for further application in animal and food industry. 


\section{Acknowledgements}

The authors appreciate Mr. Atanas Karamanov, Mr. Robert Stuski and Mr. Freddy Pezas at Department of Animal Science, University of Manitoba, for their excellent technical assistance.

\section{Authors' contributions}

SZ designed, performed the experiments and wrote this manuscript. JS contributed in doing the experiments and date analysis. $\mathrm{XQ}, \mathrm{KY}$ and $\mathrm{ML}$ were involved in modifying the manuscript. CY and CMN were major contributors in project administration and manuscript writing. All authors read and approved the final manuscript.

\section{Funding}

Not applicable.

\section{Availability of data and materials}

All raw sequences were submitted to the NCBI Sequence Read Archive (https://www.ncbi.nlm.nih.gov/sra/) under BioProject PRJNA692786. The data supporting the conclusion of this article are included in this article.

\section{Declarations}

Ethics approval and consent to participate

This study was approved by the University of Manitoba Animal Care Committee. All pigs were cared for according to the guidelines of the Canadian Council on Animal Care (CCAC, 2009).

\section{Consent for publication}

Not applicable.

\section{Competing interests}

The authors declare that they have no conflict of interest.

\section{Author details}

${ }^{1}$ College of Animal Science and Veterinary Medicine, Shenyang Agricultural University, 120 Dongling Road, Shenyang, Liaoning 110866, People's Republic of China. ${ }^{2}$ Department of Animal Science, University of Manitoba, Winnipeg, MB R3T 2N2, Canada.

Received: 5 July 2021 Accepted: 16 October 2021 Published online: 29 October 2021

\section{References}

Adam A, Levrat-Verny MA, Lopez HW, Leuillet M, Demigne C, Remesy C (2001) Whole wheat and triticale flours with differing viscosities stimulate cecal fermentations and lower plasma and hepatic lipids in rats. J Nutr 131:1770-1776

Adam CNW, Audrey SV, Paula IW (2016) The interplay between intestinal bacteria and host metabolism in health and disease: lessons from Drosophila melanogaster. Dis Model Mech 9:271-281

Brochot A, Azalbert V, LandrierJF TF, Serino M (2019) A two-week treatment with plant extracts changes gut microbiota, caecum metabolome, and markers of lipid metabolism in ob/ob mice. Mol Nutr Food Res 63:1900403

Cao Y, Liu H, Qin N, Ren X, Zhu B, Xia X (2020) Impact of food additives on the composition and function of gut microbiota: a review. Trends Food Sci Technol 99:295-310

Chen Z, Yu L, Wang X, Gu Z, Beta T (2016) Changes of phenolic profile and antioxidant activity in canaryseed (Phalaris canariensis L.) during germination. Food Chem 194:608-618

Clarissa SR, Ana CGS, Thais GM, Marcela A, Tessalia DL, Mahendra M, Ana PLD, Philippe L, Emmanuelle M, Vasco A, Ana MCF, Anderson M, Maarten G (2014) Local and systemic immune mechanisms underlying the anticolitis effects of the dairy bacterium Lactobacillus delbrueckii. PLOS ONE 9:e85923

Delzenne NM, Williams CM (2002) Prebiotics and lipid metabolism. Curr Opin Lipidol 13:61-67
Erickson KL, Hubbard NE (2000) Probiotic immunomodulation in health and disease. J Nutr 130:403S-409S

Fernando FA, Denis R, Geneviève P, Stéphanie D, Sébastien M, Thibault WV, Carole G, Quentin M, Yves D, Emile L, André M (2015) A polyphenol-rich cranberry extract protects from diet-induced obesity, insulin resistance and intestinal inflammation in association with increased Akkermansia spp. population in the gut microbiota of mice. Gut 64:872-883

Flint HJ, Scott KP, Louis P, Duncan SH (2012) The role of the gut microbiota in nutrition and health. Nat Rev Gastroenterol Hepatol 9:577-589

Frossard CP, Steidler L, Eigenmann PA (2007) Oral administration of an IL-10-secreting Lactococcuslactis strain prevents food-induced IgE sensitization. J Allergy Clin Immunol 119:952-959

Gowd V, Karim N, Shishir MRI, Chen XL, W, (2019) Dietary polyphenols to combat the metabolic diseases via altering gut microbiota. Trends Food Sci Technol 93:81-93

Hou C, Zeng X, Yang F, Liu H, Qiao S (2015) Study and use of the probiotic Lactobacillus reuteri in pigs: a review. J Anim Sci Biotechnol 6:14e9

Isaak CK, Petkau JC, Ominski KH, Rodriguez-Lecompte JC, Siow YL (2013) Seasonal variations in phenolic compounds and antioxidant capacity of Cornus stolonifera plant material: applications in agriculture. Can J Plant Sci 93:725-734

Jacobson A, Lam L, Rajendram M, Tamburini F, Honeycutt J, Pham T, Treuren WV, Pruss K, Stabler SR, Lugo K, Bouley DM, Vilches-Moure JG, Smith M, Sonnenburg JL, Bhatt AS, Huang KC, Monack D (2018) A gut commensalproduced metabolite mediates colonization resistance to Salmonella infection. Cell Host Microbe 4:296-307

Jan G, Belzacq AS, Haouzi D (2002) Propionibacteria induce apoptosis of colorectal carcinoma cells via short-chain fatty acids acting on mitochondria. Cell Death Differ 9:179-188

Kevin PR, Karen LM, Richard NF (2005) The role of enteric microflora in inflammatory bowel disease: human and animal studies with probiotics and prebiotics. Gastroenterol Clin North Am 34:465-482

Kutschera M, Engst W, Blaut M, Braune A (2011) Isolation of catechin-converting human intestinal bacteria. J Appl Microbiol 111:165-175

LeBlanc JG, Chain F, Martín R, Bermúdez-Humarán LG, Courau S, Langella P (2017) Beneficial effects on host energy metabolism of short-chain fatty acids and vitamins produced by commensal and probiotic bacteria. Microb Cell Fact 16:79

Lee JH, Valeriano VD, Shin YR, Chae JP, Kim GB, Ham JS, Chun J, Kang DK (2012) Genome sequence of Lactobacillus mucosae LM1 isolated from piglet feces. J Bacteriol 194:4766

Lick S, Drescher K, Heller KJ (2001) Survival of Lactobacillus delbrueckii subsp. bulgaricus and Streptococcus thermophilus in the terminal ileum of fistulated gottingen minipigs. Appl Environ Microbiol 67:4137-4143

Liu Z, Lin X, Huang G, Zhang W, Rao P, Ni L (2014) Prebiotic effects of almonds and almond skins on intestinal microbiota in healthy adult humans. Anaerobe 26:1-6

Makkar H, Francis G, Becker K (2007) Bioactivity of phytochemicals in some lesser-known plants and their effects and potential applications in livestock and aquaculture production systems. Animal 1:1371-1391

Mauro AD, Neu J, Riezzo G, Raimondi F, Martinelli D, Francavilla R, Indrio F (2013) Gastrointestinal function development and microbiota. Ital J Pediatr 39:15

Moorthy M, Chaiyakunapruk N, Jacob SA, Palanisamy UD (2020) Prebiotic potential of polyphenols, its effect on gut microbiota and anthropometric/clinical markers: a systematic review of randomised controlled trials. Trends Food Sci Technol 99:634-649

Moro-García MA, Alonso-Arias R, Baltadjieva M, Benítez CF, Barrial MAF, Ruisánchez ED, Santos RA, Sánchez MÁ, Miján JS, López-Larrea C (2013) Oral supplementation with Lactobacillus delbrueckii subsp. bulgaricus 8481 enhances systemic immunity in elderly subjects. Age (dordr) 35:1311-1326

Nishina PM, Freedland RA (1990) Effects of propionate on lipid biosynthesis in isolated rat hepatocytes. J Nutr 120:668-673

Ozdal T, Sela DA, Xiao J, Boyacioglu D, Chen F, Capanoglu E (2016) The reciprocal interactions between polyphenols and gut microbiota and effects on bioaccessibility. Nutrients 8:78

Pandey KB, Rizvi SI (2009) Plant polyphenols as dietary antioxidants in human health and disease. Oxid Med Cell Longev 2:270-278

Ruijschop R, Boelrijk AEM, Giffel MCT (2008) Satiety effects of a dairy beverage fermented with propionic acid bacteria. Int Dairy J 18:945-950 
Salvetti E, Torriani S, Felis GE (2012) The genus lactobacillus: a taxonomic update. Probiotics Antimicrob Proteins 4:217-226

Schafer P (2011) The Chinese medicinal herb farm: a cultivator's guide to small-scale organic herb production. Chelsea Green Publishing, White River Junction

Smits HH, Engering A, Kleij D, Jong EC, Schipper K, Capel TMM, Zaat BAJ, Yazdanbakhsh M, WierengaE A, Kooyk Y, Kapsenberg ML (2005) Selective probiotic bacteria induce $\mathrm{IL}-10$-producing regulatory $T$ cells in vitro by modulating dendritic cell function through dendritic cell-specific intercellular adhesion molecule 3-grabbing nonintegrin. J Allergy Clin Immunol 115:1260-1267

Sun Q, Shi Y, Wang F, Han D, Lei H, Zhao Y, Sun Q (2015) Study on the effects of microencapsulated Lactobacillus Delbrueckii on the mouse intestinal flora. J Microencapsul 32:669-676

Sun H, Chen Y, Cheng M, Zhang X, Zheng X, Zhang Z (2018) The modulatory effect of polyphenols from green tea, oolong tea and black tea on human intestinal microbiota in vitro. J Food Sci Technol 55:399-407

Swanson KS, Gibson GR, Hutkins R, Reimer RA, Reid G, Verbeke K, Scott KP, Holscher HD, Azad MB, Delzenne NM, Sanders ME (2020) The international scientific association for probiotics and prebiotics (ISAPP) consensus statement on the definition and scope of prebiotics. Nat Rev Gastroenterol Hepatol 17:687-701

Tong L, Wang Y, Wang Z, Liu W, Sun S, Li L, Su D, Zhang L (2016) Propionate ameliorates dextran sodium sulfate-induced colitis by improving intestinal barrier function and reducing inflammation and oxidative stress. Front Pharmacol 7:253
Torres-Pitarch A, Gardiner GE, Cormican P, Rea M, Crispie F, O'Doherty JV, Cozannet P, Ryan T, Lawlor PG (2020) Effect of cereal soaking and carbohydrase supplementation on growth, nutrient digestibility and intestinal microbiota in liquid-fed grow-finishing pigs. Sci Rep 10:1023

Wang XF, Mao SY, Liu JH, Zhang LL, Cheng YF, Jin W, Zhu WY (2011) Effect of the gynosaponin on methane production and microbe numbers in a fungus-methanogen co-culture. J Anim Feed Sci 20:272-284

Watanabe M, Kinoshita H, Nitta M, Yukishita R, Kawai Y, Kimura K, Taketomo N, Yamazaki Y, Tateno Y, Miura K, Horii A, Kitazawa H, Saito T (2010) Identification of a new adhesin-like protein from Lactobacillus Mucosae ME-340 with specific affinity to the human blood group A and B antigens. J Appl Microbiol 109:927-935

Wubben JE, Smiricky-Tjardes MR, Albin DM, Gabert VM (2001) Improved procedure and cannula design for simple-T cannulation at the distal ileum in growing pigs. Contemp Top in Lab Anim Sci 40:27-31

Yu Q, Zhu L, Wang Z, Li P, Yang Q (2012) Lactobacillus Delbrueckii Ssp. Lactis R4 prevents Salmonella Typhimurium S Pengcheng L1344-induced damage to tight junctions and adherens junctions. J Microbiol 50:613-617

\section{Publisher's Note}

Springer Nature remains neutral with regard to jurisdictional claims in published maps and institutional affiliations.

\section{Submit your manuscript to a SpringerOpen ${ }^{\circ}$ journal and benefit from:}

- Convenient online submission

- Rigorous peer review

- Open access: articles freely available online

- High visibility within the field

- Retaining the copyright to your article

Submit your next manuscript at $\boldsymbol{\nabla}$ springeropen.com 